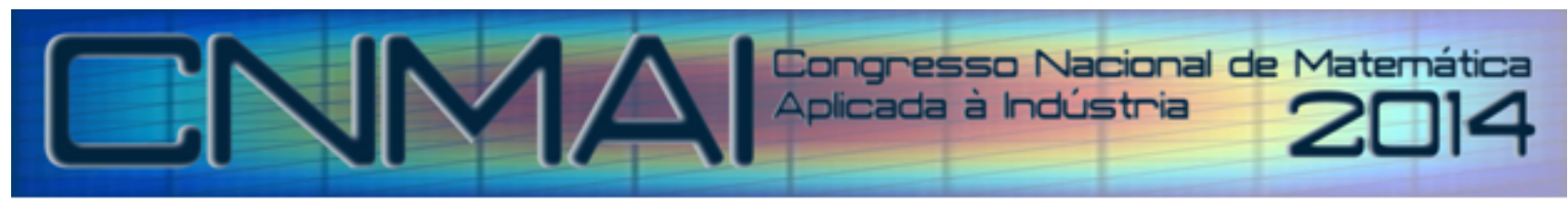

18 a 21 de novembro de 2014, Caldas Novas - Goiás

\title{
MODELAGEM COMPUTACIONAL DA INJEÇÃO DE DIÓXIDO DE CARBONO EM MEIOS POROSOS
}

\author{
Luiz Umberto Rodrigues Sica, luizsica@lncc.br ${ }^{1}$ \\ ${ }^{1}$ Grupo de Mecânica Computacional, Laboratório Nacional de Computação Científica (LNCC) \\ Av. Getúlio Vargas, 333 Quitandinha, Petrópolis, 25651, RJ, Brasil
}

\begin{abstract}
Resumo. Apresentamos uma metodologia numérica localmente conservativa para a simulação computacional do escoamento bifásico (água e $\mathrm{CO}_{2}$ ) com absorção de massa entre as fases fluidas e reação da fase $\mathrm{CO}_{2} \mathrm{com}$ a rocha em um reservatório homogêneo. Este problema é modelado por um sistema de equações diferenciais parciais basicamente composto por um subsistema parabólico para a determinação do campo de velocidades e duas equações hiperbólicas não lineares para o transporte das fases que escoam (equações da saturação e da concentração). Do ponto de vista numérico, utilizaremos a técnica de decomposição de operadores a fim de tratar apropriadamente a escala de tempo de cada fenômeno físico. Propomos a aplicação de um método de elementos finitos localmente conservativo para a velocidade da mistura e um método de volumes finitos não oscilatório de alta ordem baseado em esquemas centrais para as equações hiperbólicas não lineares que governam a saturação e a concentração das fases. Além disso, trataremos numericamente o fluxo de massa entre as fases fluidas, ou seja, a dissolução do $\mathrm{CO}_{2}$ na fase aquosa, a partir da metodologia flash que trata numericamente estas relaỗes de equilíbrio. A reação do $\mathrm{CO}_{2}$ com a rocha (precipitação), que provoca alterações na porosidade e na permeabilidade, foi tratada através da aplicação de princípios da teoria cinética.
\end{abstract}

Palavras-chave: Método de Volumes Finitos, Método de Elementos Finitos, Absorção de Massa entre as Fases, Escoamento em Meios Porosos e Sequestro Geológico de Dióxido de Carbono.

\section{INTRODUÇÃO}

$\mathrm{O}$ aquecimento global é uma realidade. Ano após ano a temperatura da Terra vem aumentando, afetando de forma preocupante o clima do planeta. Isto se deve à intensificação do efeito estufa devido à emissão de gases, decorrentes de atividades industriais. Dentre os gases que intensificam este efeito temos o dióxido de carbono $\left(\mathrm{CO}_{2}\right)$ que, segundo Ravagnani e SuslickII (2008), possui uma maior contribuição para a intensificação do efeito estufa devido à grande quantidade produzida e emitida para a atmosfera. Uma das possíveis soluções é o chamado sequestro de carbono, que consiste na separação, transporte (em estado supercrítico) e armazenamento do $\mathrm{CO}_{2}$ em reservatórios geológicos.

Modelos de sequestro geológico de $\mathrm{CO}_{2}$ devem levar em consideração escoamentos multifásicos, reações de equilíbrio e não equilíbrio e partição das fases em reservatórios. Vários autores têm estudado este problema, dentre eles: Van der Meer (1993, 1995 e 1996), Holt et al. (1995), Weir et al. (1995), Law e Bachu (1996), Lindberg (1997), Johnson et al. (2000), Ennis-King e Paterson (2002), Wellman et al. (2003), Pruess e Xu (2003), Xu e Pruess (2003) e Kumar et al. (2005), porém estes desconsideram a reação do $\mathrm{CO}_{2}$ com a rocha. Malik e Islam (2000) realizaram um estudo para o campo de Weyburn, onde o $\mathrm{CO}_{2}$ coletado foi injetado em um campo petrolífero. O objetivo foi determinar o ponto de injeção ótimo para maximizar o armazenamento de $\mathrm{CO}_{2}$ e recuperação de hidrocarbonetos. Brinks e Fanchi (2001) realizaram um estudo acoplado do movimento do $\mathrm{CO}_{2}$ e da resposta sísmica. Xu e Pruess (2003) e Pruess e Xu (2003) utilizaram um detalhado modelo geoquímico para estudar as interações entre o fluido e a rocha durante um escoamento radial. Kumar et al. (2005) realizaram uma minuciosa análise do armazenamento do $\mathrm{CO}_{2}$ utilizando um simulador comercial em uma malha de 64000 elementos. Os autores concluíram que o aprisionamento de $\mathrm{CO}_{2}$ era um mecanismo importante de armazenamento permanente. Pruess (2004) estudou o vazamento de $\mathrm{CO}_{2}$ considerando o escoamento multifásico e efeitos térmicos. Além disso, estudou o caso em que o $\mathrm{CO}_{2}$ se torna subcrítico e forma duas fases não aquosas utilizando geometria radial. Ennis-King e Paterson (2002) estudaram detalhadamente fatores que afetam o sequestro geológico de $\mathrm{CO}_{2}$ incluindo o efeito gravitacional e também utilizaram geometria radial. Outros autores consideraram os papéis da histerese da permeabilidade relativa (Spiteri et al, 2005), da dispersão (Calabrese et al, 2005) e da mistura 
convectiva (Ennis-King e Paterson, 2005) durante o armazenamento de $\mathrm{CO}_{2}$. Por fim, Obi e Blunt (2006) modelaram o armazenamento de $\mathrm{CO}_{2}$ em um aquífero no Mar do Norte utilizando o método streamline. Concluíram que a distribuição de $\mathrm{CO}_{2}$ é dominada pelo transporte advectivo devido ao fluxo multifásico e o $\mathrm{CO}_{2}$ move-se preferencialmente através dos canais de alta permeabilidade. Sem reação, o escoamento do $\mathrm{CO}_{2}$ ocorre até atingir o valor da saturação residual. A precipitação leva à diminuição da porosidade e da permeabilidade, enquanto o $\mathrm{CO}_{2}$ é armazenado na fase sólida. A eficiência de armazenamento por este mecanismo é baixa, em torno de $2 \%$, por causa da heterogeneidade do arquífero.

\section{MODELO MATEMÁTICO}

Problema: Dados os campos de compressibilidade da rocha $\beta$, o tensor de condutividade hidráulica $K$ e mobilidade da mistura fluida $\lambda_{t}$. Encontrar a pressão $p: \Omega \rightarrow R$, a velocidade de Darcy da mistura $\mathbf{v}_{D t}: \Omega \times[0, T] \rightarrow R^{n}, n=$ $1,2,3$, a porosidade $\phi: \Omega \rightarrow(0,1)$, a saturação $S_{c}: \Omega \rightarrow(0,1)$ e concentração $C: \Omega \rightarrow R$ tais que:

$$
\begin{gathered}
\mathbf{v}_{D t}=-K \lambda_{t} \nabla p \\
\beta \frac{\partial p}{\partial t}+\operatorname{div}\left(\mathbf{v}_{D t}\right)=-T_{d}^{c} \\
\frac{\partial\left(S_{c} \phi\right)}{\partial t}+\operatorname{div}\left(f_{c} \mathbf{v}_{D t}\right)=-T_{d}^{c} \\
\frac{\partial\left(C\left(1-S_{c}\right) \phi\right)}{\partial t}+\operatorname{div}\left[C\left(1-f_{c}\right) \mathbf{v}_{D t}\right]=T_{d}^{c}-T_{r} \\
\frac{\partial \phi}{\partial t}=-\left(1-\frac{m_{w}}{m_{c}}\right) T_{r} .
\end{gathered}
$$

\begin{tabular}{|c|c|}
\hline Condições de contorno & Condições iniciais \\
\hline $\mathbf{v}_{\mathbf{D t}} \cdot \mathbf{n}=q \quad$ sobre $\quad \Gamma_{N}^{v}$ & $p(x, 0)=p_{0}(x)$ \\
\hline$p=\bar{p} \quad$ sobre $\quad \Gamma_{D}^{p}$ & $\phi(x, 0)=\phi_{0}(x)$ \\
\hline$\phi=\bar{\phi} \quad$ sobre $\quad \Gamma_{D}^{\phi}$ & $S(x, 0)=S_{0}(x)$ \\
\hline$S(\mathbf{x})=s(\mathbf{x})$, sobre $\Gamma_{D}^{S}$ & $C(x, 0)=C_{0}(x)$ \\
\hline$C(\mathbf{x})=c(\mathbf{x})$, sobre $\Gamma_{D}^{C}$ & \\
\hline
\end{tabular}

onde $\Gamma=\Gamma_{N} \cup \Gamma_{D}, \Gamma_{N}^{v} \cap \Gamma_{D}^{p}=\emptyset ; f_{c}{ }^{1}$ é a função fracionária de fluxo; $T_{d}^{c}$ representa o número de mols de $C O_{2}$ na fase aquosa; $T_{r}$ representa o número de mols por unidade de volume que efetivamente reagem com a rocha; $m_{w}$ é a densidade molar da água sem $\mathrm{CO}_{2}$ dissolvido e $m_{c}$ é a densidade molar do $\mathrm{CO}_{2}$ sem água dissolvida.

\section{DISCRETIZAÇÃO DO MODELO}

No contexto de escoamentos em meios porosos, o sistema parabólico é usualmente denominado problema de Darcy ou hidrodinâmica (Eqs. 1 e 2) e o conjunto de equações hiperbólicas é constituído pela equação da saturação e pela equação da concentração (Eqs. 3 e 4). Do ponto de vista numérico, utilizaremos o fato de que a escala de tempo do problema parabólico é maior do que a do problema hiperbólico e adotaremos um algoritmo de desacoplamento no tempo.

\subsection{Evolução no tempo}

Iniciamos com a descrição do algoritmo de evolução temporal com passo fracionário associado à decomposição de operadores para a solução do sistema de equações do modelo proposto (Eqs. 1, 2, 3 e 4), ver Fig. 1. No instante de tempo inicial $t=0$, dadas as condições de contorno e iniciais, o subproblema da hidrodinâmica é resolvido para obtenção do campo de velocidades que permanece constante até o instante de tempo $t=\Delta t_{h}$. As velocidades "congeladas" são usadas nas equações de transporte convectivo que atualizam as variáveis $S_{c}$ e $C$. Neste caso, o passo de tempo $\Delta t_{c}$ é definido pela restrição CFL. Para simplificar o algoritmo faremos $\Delta t_{d}=\Delta t_{c}$ e com as novas saturações e concentrações realizamos a etapa de dissolução. Após o cômputo de todas as atualizações das variáveis $S_{c}$ e $C$ ao longo dos micropassos convectivos e de dissolução (lembrando que $\Delta t_{d}=\Delta t_{c}$ ), faremos a atualização dos campos de permeabilidade e porosidade durante o passo reativo até o instante de tempo $\Delta t_{r}$, faremos neste passo também uma simplicação no algoritmo considerando $\Delta t_{r}=\Delta t_{h}$.

\footnotetext{
${ }^{1}$ A mobilidade total (sugerida por Chavent e Roberts (1989) como função das permeabilidades relativas dos fluidos) é definida por
}

$$
\lambda_{t}=\lambda_{w}+\lambda_{c}=\frac{K_{R w}}{\mu_{w}}+\frac{K_{R c}}{\mu_{c}}
$$

onde $K_{R w}$ e $K_{R c}$ são funções escalares da saturação denominadas de coeficientes de permeabilidade relativa da água e do $C O_{2}$ com $0 \leq K_{R \beta} \leq 1$ (para $\beta=w, c$ ), parâmetros esses que quantificam a resistência adicional que um fluido exerce sobre o movimento do outro. Além disso, a função fracionária de fluxo da fase $\mathrm{CO}_{2}$ que representa a razão entre a mobilidade da fase $\mathrm{CO}_{2}$ e a mobilidade total que, segundo Chen et al. (2006), é dada por

$$
f_{c}=\frac{\lambda_{c}}{\lambda_{t}}
$$




\subsection{Hidrodinâmica}

Reformulamos o problema hidrodinâmico na sua forma mista dual com emprego de elementos de Raviart e Thomas (1977) de mais baixa ordem. Em seguida, fizemos uma condensação estática eliminando a pressão (utilizando o esquema de Euler implícito) que resulta em uma única equação a ser resolvida para a velocidade.

Formulação em velocidade: Para cada tempo $t^{m}, m \geq 1$ encontrar a velocidade $\mathbf{v}_{D t}^{m} \in U$ tal que $\forall \mathbf{v} \in U$

$$
\int_{\Omega} k^{-1} \lambda_{t}^{-1} \mathbf{v}_{D t} \cdot \mathbf{v} d \Omega+\frac{\Delta t}{\beta} \int_{\Omega} \operatorname{div} \mathbf{v}_{D t} \operatorname{div} \mathbf{v} d \Omega=\int_{\Omega} P^{m-1} \operatorname{div} \mathbf{v} d \Omega-\frac{\Delta t}{\beta} \int_{\Omega}\left(T_{d}^{c}\right)^{m} d i v \mathbf{v} d \Omega-\int_{\Gamma_{D}^{p}} \bar{p} \mathbf{v} \cdot \mathbf{n} d \Gamma
$$

Para maiores detalhes no desenvolvimento desta formulação posta unicamente em função do campo de velocidades, ver trabalhos de Raviart e Thomas (1977), Douglas Jr et al. (1983), Correa e Loula (2007) e Correa e Loula (2008).

\subsection{Problema preditor}

Dados os campos de porosidade $\phi(\mathbf{x})$ e da velocidade de Darcy da mistura $\mathbf{v}_{D t}$ no istante $t^{n}$, encontrar a saturação $S$ tal que

$$
\phi \frac{\partial S}{\partial t}+\operatorname{div}(\mathbf{f})=0 \quad \text { em } \quad \Omega \times\left(t^{n}, t^{n+1}\right)
$$

onde $\mathbf{f}(S)=\left[\frac{f(S)}{g(S)}\right]$ e satisfazendo a condição inicial $S\left(\mathbf{x}, t^{n}\right)=S^{n}$.

O método KT proposto por Kurganov e Tadmor (2000) foi desenvolvido para leis de conservação não lineares da forma da Eq. (9), para um campo de porosidades $\phi$ constante. A extensão da formulação para o caso onde $\phi=\phi(\mathbf{x})$, ou seja, para o caso em que a porosidade é função da posição, foi desenvolvida por Correa e Borges (2013). Tal formulação é de suma importância para a simulação de meios porosos, sua construção é baseada no algoritmo REA descrito a seguir

\section{Algoritmo REA}

- Reconstrução: A partir dos valores computados da média $S_{i, j}^{n}$ e das aproximações das derivadas $\left(S_{x}\right)_{i, j}^{n}$ e $\left(S_{y}\right)_{i, j}^{n}$ obtidas utilizando os limitadores MinMod, reconstruiremos a aproximação polinomial por partes que, para o caso bidimensional, é um interpolante linear por partes dado por:

$$
L_{i, j}^{n}(\mathbf{x})=S_{i, j}^{n}+\left(S_{x}\right)_{i, j}^{n}\left(x-x_{i}\right)+\left(S_{y}\right)_{i, j}^{n}\left(y-y_{j}\right), \quad \text { para } \quad \mathbf{x} \in C_{i, j} .
$$

- Evolução: Evoluiremos a equação hiperbólica no tempo com condições iniciais no tempo $t^{n}$, a fim de obter a média $S_{D_{i, j}}^{n+1}$ no tempo $t^{n+1}$ em uma malha dual. É de suma importância a utilização de informações sobre o valor das velocidades locais na definição do tamanho da malha dual, que caracterizará a largura dos leques de Riemann.

- Projeção: Reprojetaremos a média em malha dual $S_{D_{i, j}}^{n+1}$ de cada célula na malha original $\tau_{h}$, a fim de obtermos a média em cada célula na malha original no tempo $t^{n+1}$.

Aplicando esta metodologia podemos reescrever a formulação em termos dos fluxos numéricos através da expressão

$$
\frac{d S_{i, j}}{d t}=\frac{H_{r}-H_{l}}{\Delta x}+\frac{H_{u}-H_{d}}{\Delta y}
$$

onde os fluxos numéricos são dados por

$$
\begin{aligned}
& H_{\beta}=\frac{a_{\beta} \phi_{\beta}}{\phi_{c}+\phi_{\beta}}\left(S_{\beta}^{+}-S_{\beta}^{-}\right)-\frac{1}{\phi_{c}}\left[\frac{\phi_{c} \varphi\left(S_{\beta}^{+}\right)+\phi_{\beta} \varphi\left(S_{\beta}^{-}\right)}{\phi_{c}+\phi_{\beta}}\right], \operatorname{para} \beta=r, u \\
& H_{\beta}=\frac{a_{\beta} \phi_{\beta}}{\phi_{c}+\phi_{\beta}}\left(S_{\beta}^{+}-S_{\beta}^{-}\right)-\frac{1}{\phi_{c}}\left[\frac{\phi_{\beta} \varphi\left(S_{\beta}^{+}\right)+\phi_{c} \varphi\left(S_{\beta}^{-}\right)}{\phi_{c}+\phi_{\beta}}\right], \operatorname{para} \beta=l, d
\end{aligned}
$$

$\operatorname{com} \varphi=f \operatorname{para} \beta=r, l$ e $\varphi=g$ para $\beta=u, d$.

\subsection{Problema corretor}

Descreveremos a formulação do passo corretor sem perda de generalidade para a equação da saturação. Porém, este passo também será calculado para a equação da concentração.

Conhecidos a solução obtida no passo preditor $S^{*(n+1)}$ e o campo $\phi(\mathbf{x}, t)$, achar $S_{c}^{* *}\left(\mathbf{x}, t_{n+1}\right)$ para cada subintervalo de tempo $\Delta t_{c}=\left(t_{n}, t_{n+1}\right), n=0, \ldots, N-1 \operatorname{com} t_{0}=0, t_{n}=T$, tal que

$$
\phi \frac{\partial S_{c}^{* *}}{\partial t}=-S_{c}^{* *} \frac{\partial \phi}{\partial t}
$$

sujeito à condição inicial

$$
S_{c}^{* *}\left(x, t_{n}\right)=S_{c}^{*(n+1)} .
$$


Integrando a equação acima no intervalo $\Delta t_{c}$ para cada ponto $x \in \Omega$, obtemos

$$
S^{* * n+1}=\frac{\phi^{n} S^{* n+1}}{\phi^{n+1}}
$$

\subsection{Tratamento do termo de fonte de dissolução}

Segundo Obi e Blunt (2006), para computar o número de moles de $C_{2}$ na fase líquida $T_{d}^{c}$ e atualizar os valores da saturação $S_{c}$ e da concentração $C$ no tempo $t^{n+1}$, podemos utilizar o algoritmo

$$
\left(T_{d}^{c}\right)^{n+1}=\phi^{n+1}\left[\left(1-S_{c}^{n+1}\right) C^{n+1}+m_{c} S_{c}^{n+1}\right]
$$

onde

$$
\begin{aligned}
& C^{n+1}=K_{d} \quad \text { e } \quad S_{c}^{n+1}=\frac{\frac{\left(T_{d}^{c}\right)^{n+1}}{\phi^{n+1}}-K_{d}}{m_{c}-K_{d}} \quad \text { se } \quad\left(T_{d}^{c}\right)^{n+1} \geq \phi^{n+1} K_{d} \\
& C^{n+1}=\frac{\left(T_{d}^{c}\right)^{n+1}}{\phi^{n+1}} \quad \text { e } \quad S_{c}^{n+1}=0 \quad \text { se } \quad\left(T_{d}^{c}\right)^{n+1}<\phi^{n+1} K_{d} .
\end{aligned}
$$

A constante de dissolução é definida como

$$
K_{d}=\frac{x_{\mathrm{CO}_{2}}^{L} m_{w}}{1-x_{C O_{2}}^{L}\left(1-\frac{m_{w}}{m_{c}}\right)} .
$$

onde $m_{w}$ é a densidade molar da água sem $\mathrm{CO}_{2}$ dissolvido, $m_{c}$ é a densidade molar do $\mathrm{CO}_{2}$ sem água dissolvida e $x_{\mathrm{CO}}^{L}$ representa a fração molar de $\mathrm{CO}_{2}$ que pode se dissolver na fase aquosa.

\subsection{Tratamento do termo de fonte de reação}

Ao aplicarmos o algoritmo desenvolvido por Obi e Blunt (2006) para resolução da Eq. (5), computaremos o termo de fonte de reação $\left(T_{r}\right)$ e atualizaremos o campo de porosidade $\phi$. Além disso, será utilizada uma lei empírica para a atualização do campo de permeabilidade $K$.

Segundo Obi e Blunt (2006), para computar o número total de moles de $\mathrm{CO}_{2}$ por unidade de volume em ambas as fases $\left(m_{t t}\right)$, podemos utilizar o seguinte algoritmo:

$$
m_{\mathrm{tt}}=\phi^{n}\left[\left(1-S_{c}^{n}\right) C^{n}+m_{c} S^{n}\right] .
$$

O número total de moles de $\mathrm{CO}_{2}$ por unidade de volume que efetivamente reage com a rocha é definido como

$$
T_{r}=\frac{m_{w}}{1-\frac{m_{w}}{m_{c}}} .
$$

Para calcularmos a variação da porosidade, utilizaremos o seguinte procedimento:

$$
\begin{aligned}
& \phi^{n+1}=\phi^{n}\left[\exp \left(-K \Delta t_{r}\right)\right] \quad \text { se } \quad m_{t t} \geq T_{r} \phi^{n}\left[1-\exp \left(-K \Delta t_{r}\right)\right] \\
& \phi^{n+1}=\phi^{n}-\frac{m_{t t}}{T_{r}} \quad \text { se } \quad m_{t t}<T_{r} \phi^{n}\left[1-\exp \left(-K \Delta t_{r}\right)\right] .
\end{aligned}
$$

Por fim, a permeabilidade será atualizada a partir da formulação empírica (Wellman et al. (2003))

$$
K^{n+1}=K^{n}\left(\frac{\phi^{n+1}}{\phi^{n}}\right)^{3.4}
$$

\section{RESULTADOS NUMÉRICOS}

Realizamos inicialmente da forma mais próxima possível o mesmo experimento de Obi e Blunt (2006). Logo após, estudamos o efeito dos fenômenos de dissolução e reação sobre o movimento de uma pluma de $\mathrm{CO}_{2}$ em um meio poroso homogêneo. O campo de velocidade foi calculado a partir da resolução do subproblema da hidrodinâmica (Eqs. 1 e 2), utilizando o método de elementos finitos misto. Aplicamos o método KT (versão modificada por Correa e Borges (2013) para incluir a porosidade variável) na resolução do transporte (Eqs. 3 e 4). O cômputo dos termos de fonte referentes à dissolução do $\mathrm{CO}_{2}$ na fase água foi feito utilizando o algoritmo descrito na subseção (3.5) e, os campos de porosidade e permeabilidade, foram atualizados fazendo uso do algoritmo reativo descrito em (3.6).

Para os experimentos que seguem, consideramos um reservatório aproximadamente incompressível utilizando um valor para $\beta$ (compressibilidade da rocha) da ordem de $10^{-9}$. 


\subsection{Comparando resultados com a literatura}

O objetivo das seguintes simulações é realizar da forma mais próxima possível o mesmo experimento de Obi e Blunt (2006). Faremos um estudo qualitativo, tendo em vista que algumas informações sobre as simulações não puderam ser encontradas no referido artigo. Além disso, cabe ressaltar que em suas simulações, Obi e Blunt (2006) utilizaram o método de streamline para resolver o transporte.

Consideramos os seguintes parâmetros físicos: temperatura de $353 K$, densidade da água de $1050 \mathrm{~kg} . \mathrm{m}^{-3}$, densidade do $\mathrm{CO}_{2}$ de $710 \mathrm{~kg} . \mathrm{m}^{-3}$, viscosidade da água de $5 \times 10^{-4} \mathrm{~Pa}$.s, viscosidade do $C \mathrm{O}_{2}$ de $6 \times 10^{-5} \mathrm{~Pa} . \mathrm{s}$, densidade molar do $\mathrm{CO}_{2}\left(m_{c}\right)$ de 16140 moles.m ${ }^{-3}$, densidade molar efetiva do sólido $\left(T_{r}\right)$ de 26500 moles.m ${ }^{-3}$, porosidade de $\phi=0,15$, permeabilidade absoluta de $K=100 \mathrm{mD}$, saturação residual da água de $S_{r w}=0,2$, saturação residual do $C O_{2}$ de $S_{r c}=0,2$. Utilizamos um reservatório de dimensões $2000 \mathrm{~m} \times 1500 \mathrm{~m} \times 200 \mathrm{~m}$ e uma malha de $400 \times 1 \times 1$ elementos. Adotamos o ponto de injeção a 750m a partir da origem, a pressão neste ponto é de $28 \mathrm{MPa}$ e no bordo direito de $0 \mathrm{MPa}$.

Injetamos $\mathrm{CO}_{2}$ durante 20 anos à uma vazão volumétrica de $0,18 \frac{\mathrm{m}}{\mathrm{ano}}$.

Tabela 1. Dados do problema unidimensional.

\begin{tabular}{|l|l|}
\hline saturação inicial & $S_{c}(\mathbf{x}, 0)=0$ \\
\hline condição de contorno da velocidade & $\mathbf{v}_{D t}(0, t)=0,18 \frac{\mathrm{m}}{\mathrm{ano}}$ \\
\hline $\begin{array}{l}\text { condição de contorno da saturação } \\
\text { (injeção) }\end{array}$ & $S_{c}(0, t)=0,8$ \\
\hline
\end{tabular}

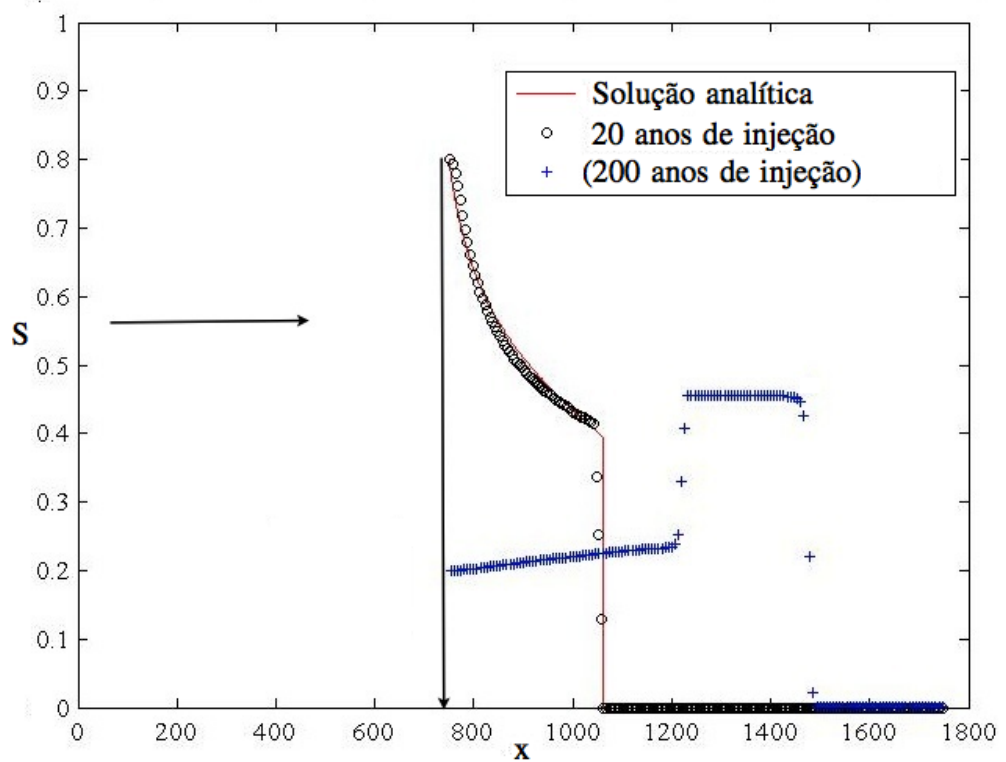

(a)

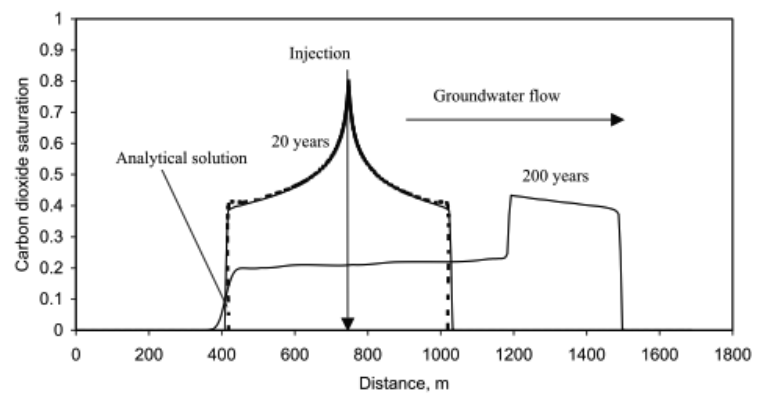

(b)

Figura 1. (a) Simulação unidimensional do caso inerte utilizando o método KT. (b) Solução obtida por Obi e Blunt (2006) para o caso inerte.

Observamos que qualitativamente nossos resultados são bastante similares aqueles encontrados por Obi e Blunt (2006) em ambos os casos. Percebemos um atraso na frente de onda em 200 anos ao considerarmos a dissolução (Fig. 2a e Fig. 


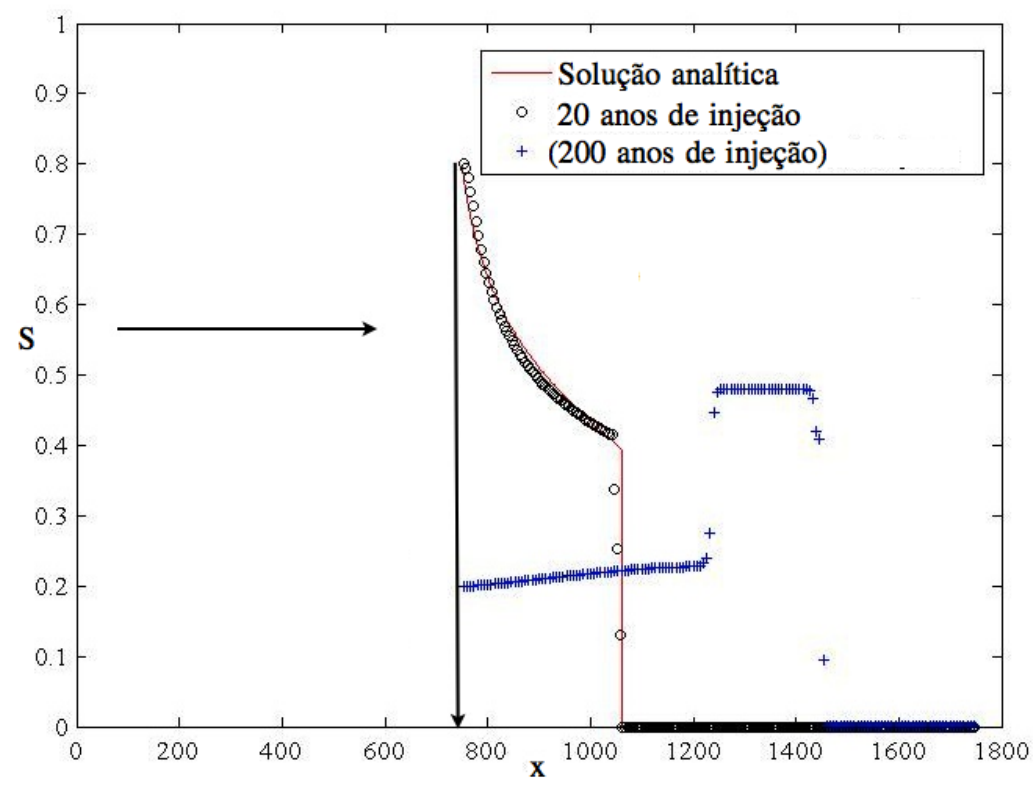

(a)

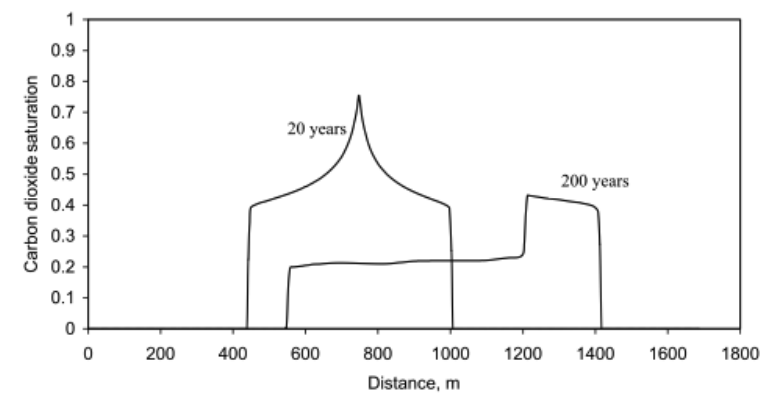

(b)

Figura 2. (a) Simulação unidimensional considerando o efeito da dissolução utilizando o método KT. (b) Solução obtida por Obi e Blunt (2006) considerando o efeito de dissolução.

2b) quando comparado ao caso inerte (Fig. 1a e Fig. 1b). Isto é devido ao fato de parte do $\mathrm{CO}_{2}$ ter sido transferido para a fase aquosa como soluto.

Por fim, além da dissolução do $\mathrm{CO}_{2}$ como soluto na fase água, consideramos a reação do $\mathrm{CO}_{2}$ com a rocha.

Observamos que durante a fase de injeção (Fig. 3a e Fig. 3b), o perfil de saturação sofreu um atraso (com concentrações menores) em comparação à primeira simulação (Fig. 2a e Fig. 2b), pois parte do $\mathrm{CO}_{2}$ precipitou. No entanto, depois de 200 anos o impacto sobre o perfil de saturação é dramático, tendo em vista que houve tempo suficiente para uma quantidade significativa de precipitação e a reação causou uma queda na saturação do $C \mathrm{O}_{2}$ abaixo do valor da saturação residual.

Apesar de não conseguirmos simular exatamente o mesmo problema de Obi e Blunt (2006), observamos que o comportamento das soluções são qualitativamente semelhantes, indicando que os métodos propostos se aproximam corretamente do problema.

\subsection{Meio homogêneo}

Agora estudaremos o efeito da dissolução e da reação em uma simulação bidimensional. Para isso, estudaremos uma pluma de $\mathrm{CO}_{2}$ em um meio poroso homogêneo, sendo que a mesma irá se propagar apenas devido à ação do campo de velocidades.

Os dados iniciais assim como as condições de contorno estão representados na Tab. (2) e na Fig. 4. 


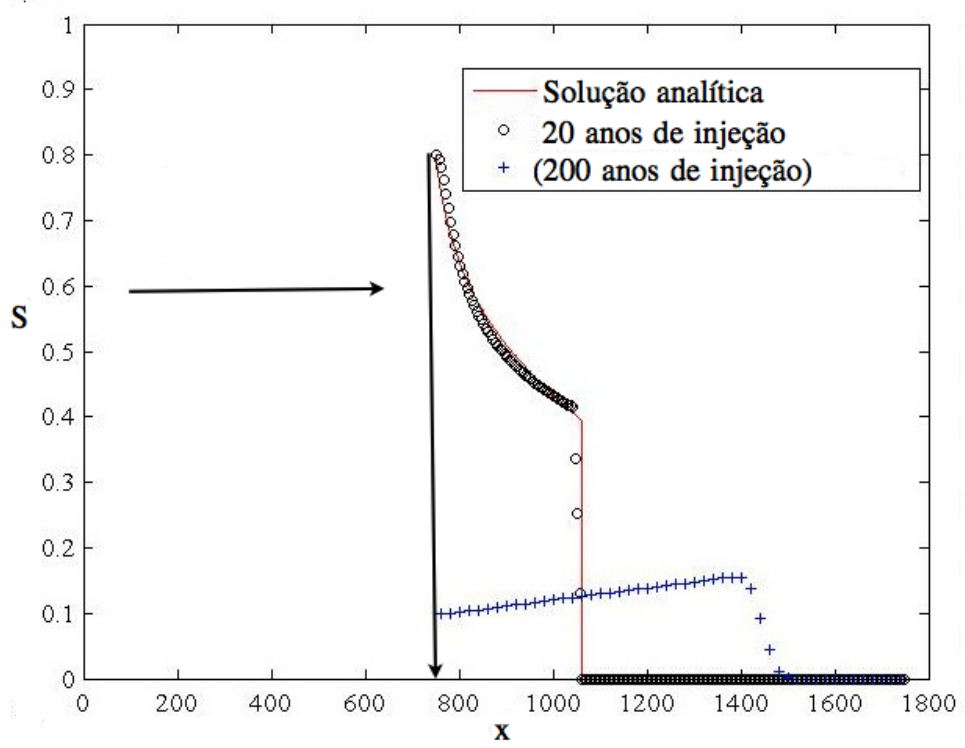

(a)

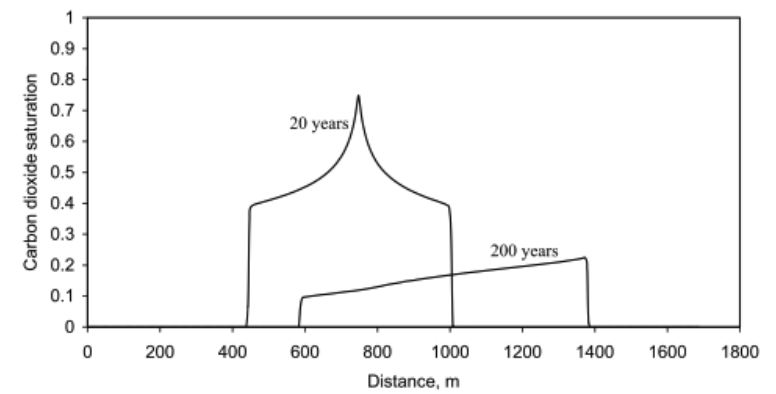

(b)

Figura 3. (a) Simulação unidimensional considerando os efeitos da dissolução e da reação utilizando o método KT. (b) Solução obtida por Obi e Blunt (2006).

Tabela 2. Dados do problema bidimensional.

\begin{tabular}{|l|l|}
\hline Descrição & Dados \\
\hline dimensão do domínio & {$[0,1] \times[0,1]$} \\
\hline malha adotada & $150 \times 150 \mathrm{c} \tilde{\mathrm{A}}(\mathrm{C})$ lulas \\
\hline porosidade inicial & $\phi=0,25$ \\
\hline permeabilidade absoluta & $K=100 \mathrm{mD}$ \\
\hline compressibilidade da rocha & $\beta=5 \times 10^{-9}$ \\
\hline pressão inicial & $p_{0}=0 \mathrm{MPa}$ \\
\hline velocidade de percolação total & $\mathbf{v}_{D t}=[1,0]^{T}$ \\
\hline saturação inicial & $S(\mathbf{x}, 0)=0$ \\
\hline saturação residual da $\tilde{A}_{i}$ gua & $S_{r w}=0,1$ \\
\hline saturação residual do $C O_{2}$ & $S_{r c}=0,1$ \\
\hline permeabilidade relativa da água & $K_{r w}=\frac{\left(1-S-S_{r w}\right)^{2}}{\left(1-S_{r w}\right)^{2}}$ \\
\hline permeabilidade relativa do $C O_{2}$ & $K_{r c}=\frac{\left(S-S_{r c}\right)^{2}}{\left(1-S_{r c}\right)^{2}}$ \\
\hline condição de contorno (injeção) & $S(\mathbf{x}, t)=0,8$ sobre $\Gamma_{I n j}$ enquanto \\
& $n \triangle t_{c}<30000 s$ \\
\hline $\begin{array}{l}\text { condição de contorno da velocidade } \\
\text { (hidrodinâmica) }\end{array}$ & $\mathbf{v}_{D t}=1,0$ sobre $\Gamma$, onde $\Gamma=$ \\
\hline tempo total de simulação & $\Gamma_{1 a} \cup \Gamma_{I n j} \cup \Gamma_{1 b}$ \\
\hline
\end{tabular}

Simulamos para as mesmas condições iniciais e de contorno dois casos: o primeiro desconsiderando a dissolução do $\mathrm{CO}_{2}$ na fase água e a reação com a fase sólida, que será chamado de caso inerte; e o segundo considerando os fenômenos de dissolução e reação. 


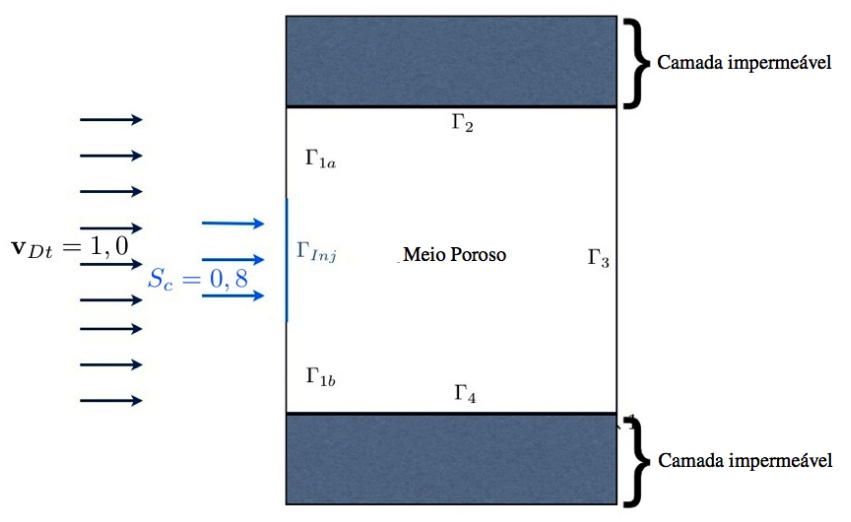

Figura 4. Condições de contorno.

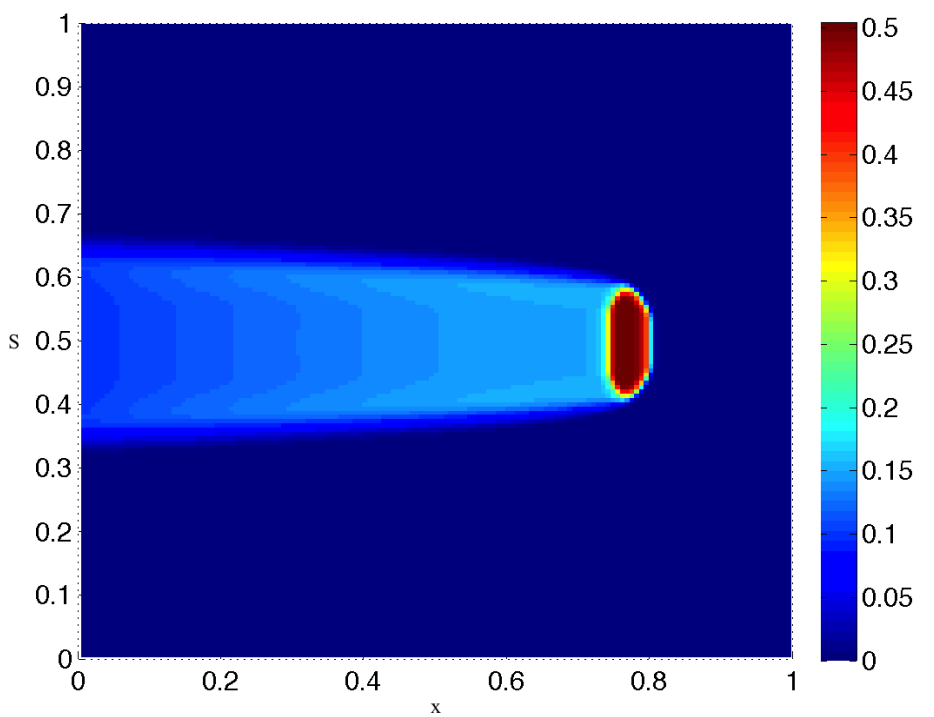

(a)

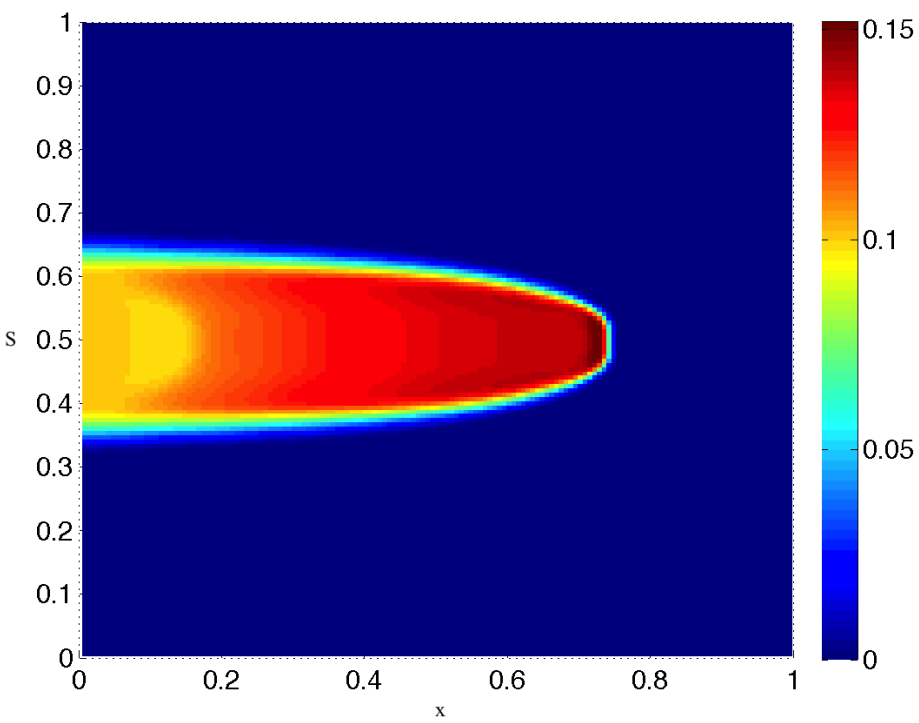

(b)

Figura 5. Para um tempo total de simulação $t=110000$ s e distância $x=1 \mathrm{~km}$ : (a) Caso inerte. (b) Considerando dissolução e reação do $\mathrm{CO}_{2}$. 
Para as mesmas condições iniciais e de contorno, ao considerarmos os fenômenos de dissolução e reação na simulação, observamos a absorção da pluma de $\mathrm{CO}_{2}$ pela fase água e sua reação com a rocha (precipitação) conforme ilustrado (Fig. 3a e Fig. 3b).

\section{REFERÊNCIAS}

Brinks, J. e Fanchi, J. 2001. Geological sequestration: Modeling and monitoring injected $\mathrm{CO}_{2}$. Soc. of Pet. Eng., SPE Pap. 66749.

Calabrese, M., Masserano, F. e Blunt, M. J. 2005. Simulation of physical-chemical processes during carbon dioxide sequestration in geological structures. SPE Pap. 95820, Soc. of Pet. Eng., Richardson, Tex.

Chavent, G. e Roberts, J. 1989. A unified physical presentation of mixed, mixed-hybrid finite elements and usual finite differences for the determination of velocities in waterflow problems. Rapports de Recherche, INRIA - Institut National de Recherche en Informatique et en Automatique.

Chen, Z., Huan, G. e Ma, Y. 2006. Computational methods for multiphase flows in porous media. Computational Science and Engineering. Society for Industrial and Applied Mathematics.

Correa, M. R. e Borges, M. R. 2013. A semidiscrete central scheme for scalar hyperbolic conservation laws with heterogeneous storage coefficient and its application to porous media flow. International Journal for Numerical Methods in Fluids, 1-19.

Correa, M. R. e Loula, A. F. D. 2007. Stabilized velocity post-processings for Darcy flow in heterogeneous porous media. Communications in Numerical Methods in Engineering, 23:461-489.

Correa, M. R. e Loula, A. F. D. 2008. Unconditionally stable mixed finite element methods for Darcy flow. Computer Methods in Applied Mechanics and Engineering, 197:1525-1540.

Douglas Jr., J., Ewing, R. E. e Wheeler, M. F. 1983. The approximation of the pressure by a mixed method in the simulation of miscible displacement. RAIRO Analyse Numériquel Numerical Analysis, 17:17-33.

Ennis-King, J. e Paterson, L. 2002. Engineering aspects of geological sequestration of carbon dioxide. Soc. of Pet. Eng., SPE Pap. 77809.

Ennis-King, J. e Paterson, L. 2005. Role of convective mixing in the long-term storage of carbon dioxide in deep saline aquifers. SPE J.. 10(3), 349â356.

Holt, T., Jensen, J. e Lindeburg, E. 1995. Underground storage of $\mathrm{CO}_{2}$ in aquifers and oils reservoir. Energy Convers. Manage, 36(6-9):535-538.

Johnson, J., Steefel, J., Nitao, J. e Knauss, K. 2000. Reactive transport modeling of subsurface $\mathrm{CO}_{2}$ sequestration: Identication of optimal target reservoir and evaluation of performance based on geochemical, hydrologic and structural constraints. 8th International Forum, Int. Energy Found., Las Vegas, Nev., 23-28.

Kumar, A., Ozah, M., Pope, G., Bryant, S., Sepehmoori, K., e Lake, L. 2005. Reservoir simulation of $\mathrm{CO}_{2}$ storage in deep saline aquifers. SPE J., 10(3):336-348.

Kurganov, A. e Tadmor, E. 2000. New High-Resolution Central Schemes for Nonlinear Conservation Laws and Convection-Diffusion Equations. Journal of Computational Physics, 160(1):241-282.

Law, D. e Bachu, S. 1996. Hydrogeological and numerical analysis of $\mathrm{CO}_{2}$ disposal in deep sedimentary aquifers in the alberta sedimentary basin. Energy Convers. Manage, 37(6-8):1167-1174.

Lindberg, E. 1997. Escape of $\mathrm{CO}_{2}$ from aquifers. Energy Convers. Manage, 38:S235-S240.

Malik, Q. e Islam, M. 2000. $\mathrm{CO}_{2}$ injection in the weyburn field of canada: Optimization of enhanced oil recovery and greenhouse gas storage with horizontal wells. Soc. of Pet. Eng., SPE Pap. 59327.

Obi, E. I. e Blunt, M. J. 2006. Streamline-based simulation of carbon dioxide storage in a north sea aquifer. Water Resources Research, 42(3):1-13.

Pruess, K. e Xu, J. 2003. Numerical modeling of aquifer disposal of $\mathrm{CO}_{2}$. SPE.

Pruess, K. 2004. Numerical simulation of $\mathrm{CO}_{2}$ leakage from a geologic disposal reservoir, including transitions from a super-to subcritical conditions, and boiling of liquid $\mathrm{CO}_{2}$. SPE J., (9):237-248.

Ravagnani, A. e SuslickII, S. 2008. Modelo dinâmico de sequestro geológico de $\mathrm{CO}_{2}$ em reservatórios de petróleo. Revista Brasileira de Ciências.

Raviart, P. A. e Thomas, J. M. 1977. A mixed finite element method for second order elliptic problems. Math. Aspects of The F.E.M., 292-315.

Spiteri, E. J., Juanes, R., Blunt, M. J. e Orr Jr, F. M. 2005. Relative permeability hysteresis: Trapping models and application to geological $\mathrm{CO}_{2}$ sequestration. SPE Pap. 96448, Soc. of Pet. Eng., Richardson, Tex.

Van der Meer, L. 1993. The conditions limiting $\mathrm{CO}_{2}$ storage in aquifers. Energy Convers. Manage, 34(959-966).

Van der Meer, L. 1995. The $\mathrm{CO}_{2}$ storage efficiency in aquifers. Energy Convers. Manage.

Van der Meer, L. 1996. Computer modelling of underground $\mathrm{CO}_{2}$ storage. Energy Convers. Manage.

Weir, G., White, S. e Kissling, W. 1995. Reservoir storage and containment of greenhouse gases. Energy Convers. Manage, 36(6-9):531-534.

Wellman, T., Grigg, R., McPherson, B., Svec, R., e Lichtner, P. 2003. The evaluation of $\mathrm{CO}_{2}$ brine-rock interaction with 
laboratory flow test and reactive transport modeling. Soc. of Pet. Eng., SPE Pap. 80228.

$\mathrm{Xu}$, J. e Pruess, K. 2003. Reactive geochemical transport simulation to study mineral trapping for $\mathrm{CO}_{2}$ disposal in deep saline arenaceous aquifers. J. Geophys. Res., 108(B2).

\title{
COMPUTATIONAL MODELING OF THE INJECTION OF CARBON DIOXIDE IN POROUS MEDIA
}

\author{
Luiz Umberto Rodrigues Sica, luizsica@lncc.br ${ }^{1}$ \\ ${ }^{1}$ Grupo de Mecânica Computacional, Laboratório Nacional de Computação Científica (LNCC) \\ Av. Getúlio Vargas, 333 Quitandinha, Petrópolis, 25651, RJ, Brasil
}

\begin{abstract}
We present a locally conservative numerical methodology to simulate the two-phase flow (water and $\mathrm{CO}_{2}$ ) with mass absorption between the fluid phases and reaction between the $\mathrm{CO}_{2}$ phase and rock in a homogeneous reservoir. This problem is modeled by a system of partial differential equations, which basically consists in a parabolic subsystem for determining the velocity field and two non-linear hyperbolic equations for the transport of phases that flow (equations of saturation and concentration). From the numerical point of view, we use the operator splitting technique to properly treat the time scale of each physical phenomenon. We propose the application of a locally conservative finite element method for the total Darcy velocity and a high-order non-oscillatory central-scheme finite volume method for nonlinear hyperbolic equations that govern the saturation and concentration of phases. Furthermore, we treat numerically the mass flux between fluid phases, the dissolution of $\mathrm{CO}_{2}$ in the aqueous phase, using the flash methodology that treats numerically equilibrium reactions. The reaction of $\mathrm{CO}_{2}$ with rock (precipitation), which causes changes in porosity and permeability, was treated by applying principles of kinetic theory.
\end{abstract}

Keywords: Finite Volume Method, Finite Element Method, Mass Absorption Between Phases, Flow in Porous Media, Geologic Sequestration of Carbon Dioxide. 